Editorial

\title{
Rapidly progressing juvenile scleroderma: A case report
}

\author{
Shah Z ${ }^{1}$, Mohan D.S. ${ }^{2}$, Parikh Y. ${ }^{3}$ \\ ${ }^{1}$ Dr. Zalak Shah, Assistant Professor, ${ }^{2}$ Dr. Dhanya Soodhana Mohan, Junior Resident, ${ }^{3}$ Dr. Yogesh Parikh, Head and \\ Professor; all authors are attached with Department of Pediatrics, PDU Medical College, Rajkot.
}

Corresponding Author: Dr. Dhanya Soodhana Mohan, Junior Resident, Department of Pediatrics, PDU Medical College, Rajkot, Email: dhanyasoodhana@gmail.com

\begin{abstract}
Juvenile Scleroderma encompasses a range of conditions unified by the presence of fibrosis of the skin. Juvenile scleroderma is of two major types: Juvenile localized scleroderma and Juvenile Systemic sclerosis. Juvenile localized scleroderma is the predominant type seen in children $(>95 \%)$. We report a case of systemic sclerosis in a child, which is associated with higher mortality and morbidity. Apart from the characteristic mask like face, arthritis was the predominant complaint in our case.
\end{abstract}

Keywords: Juvenile systemic sclerosis, Arthritis, Juvenile Scleroderma

\section{Introduction}

The term scleroderma literally means sclerosing or hardening of the skin. "Scleroderma" encompasses both forms of the disease: Systemic sclerosis (SSc), is characterized by the fibrosis of the skin, vascular and visceral organs and is more common among the adults and localized scleroderma (LS), is characterized by fibrosis of skin and underlying tissue without vascular or internal organ involvement, is more common amongst children. They both share a common underlying pathophysiology of excessive collagen deposition in an autoimmune setting. Even then these two entities are clinically different with unique prognosis and morbidities [1]. The approximate female to male ratio of pediatric SSc is $4: 1$ and for pediatric LS is $2: 1[2,3,4]$.

The various organ systems involved in juvenile SSc are skin, GIT, respiratory, renal, musculoskeletal and cardiovascular. The pattern of organ involvement is the same in juvenile SSC as in the adult SSc patient [5].

\section{Case Report}

An eleven year old girl presented to our tertiary care centre with the complaints of painful movements of the joints of the hand and stiffness of the joints since 6 months. The child was apparently normal 6 months back when she started developing pain in the joints starting with the joints of the hand, wrist and elbow which later involved the ankle and the knee joints. The above symptoms had increased one month prior to the admission and the child had difficulty in walking. The movements were painful; she had developed proximal muscle weakness since one month and was unable to attend school. The mother had noted that the stiffness was more in the morning and eased out during the day. There was no history of similar complaints in the family. Child was pale and thin built. All the vitals were normal. She had sparse hair, shiny stretched skin, the face appeared mask like and had a beaked nose and mouth opening was restricted $(2.5 \mathrm{~cm})$.

The child also had hypopigmented macules and patches bilaterally over the temporal region. Sclerodactyly and stellate scars were observed on the finger tips. Flexion deformity was present in all the fingers at the proximal and distal interphalangeal joints. The child also had restriction of movements at the elbows and shoulder. The movements at the knee were also restricted and painful. Cardiovascular system, respiratory and the central nervous system examination were normal. The ophthalmic examination revealed dryness of the eyes and no other significant finding was present. Orthopedic reference was also done and the child was started on physiotherapy. Raynaud's phenomenon was negative.

Manuscript received: $30^{\text {th }}$ May 2018

Reviewed: $8^{\text {th }}$ June 2018

Author Corrected: $15^{\text {th }}$ June 2018

Accepted for Publication: $20^{\text {th }}$ June 2018 
Editorial

Investigations revealed hemoglobin of $10.1 \mathrm{~g} / \mathrm{dl}$, total count of $14300 / \mathrm{mm}^{3}$ and a platelet count of 5.4 lakh. The differential count included $74 \%$ neutrophils, $23 \%$ lymphocytes, $02 \%$ monocytes and $01 \%$ eosinophils. The erythrocyte sedimentation rate was $80 \mathrm{~mm} / \mathrm{hr}$. Urine routine and microscopy was normal. The anti- streptolysin $\mathrm{O}$ factor, C- reactive protein and Rheumatoid Arthritis factor were negative. The chest X ray and Ultrasonography of the abdomen showed no abnormality. The renal function test and liver function test of this child was within normal limits. ANA profile done by immunodot method was positive. It showed specific bands for Scl- 70 antibodies. ECG and 2D Echocardiography done were within normal limits, with no pulmonary hypertension. Pulmonary function test done showed severe restrictive type of pattern. Skin biopsy was done and revealed increased collagen bundles.

Thus, confirming the diagnosis of Systemic Sclerosis, the patient was started on a weekly regimen of methotrexate, 15 $\mathrm{mg} / \mathrm{m}^{2}$ as a single oral dose as a therapy for the skin involvement. The child was given pulse methyl prednisolone therapy for 3 days a month for three months. The child was later continued on oral low dose prednisolone $(0.2-0.4 \mathrm{mg} / \mathrm{kg} / \mathrm{day})$ which was gradually tapered. Prednisolone helped treat the skin involvement, pulmonary fibrosis and musculoskeletal involvement (myositis, arthritis, and tenosynovitis). Two weeks later on follow up, patient had an improved complete blood picture and there was a marked reduction in the pain swelling of the joints. The child had marked improvement at the end of three months.

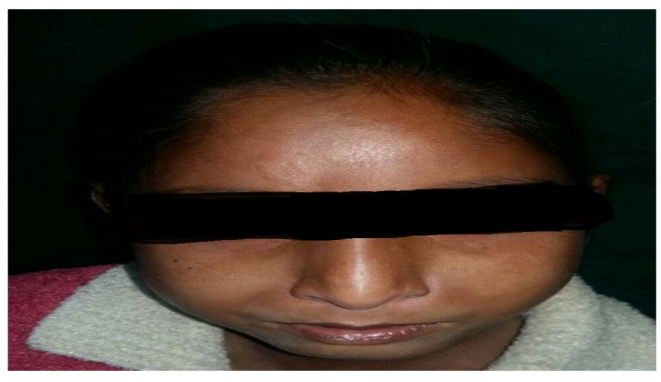

Picture 1: The mask like appearance of the face and the beaked nose in the child

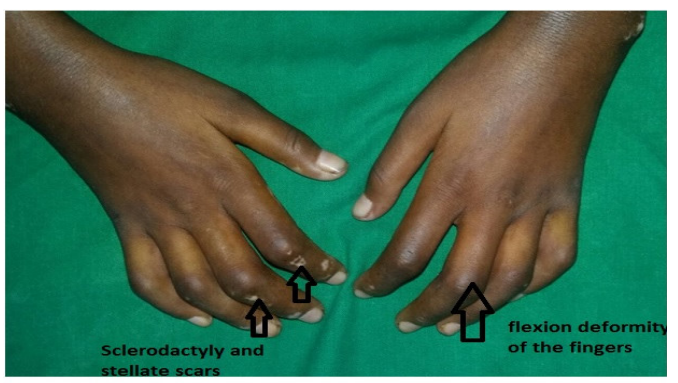

Picture 2: Sclerodactyly and stellate scars on the finger tips and flexion deformity of all the fingers

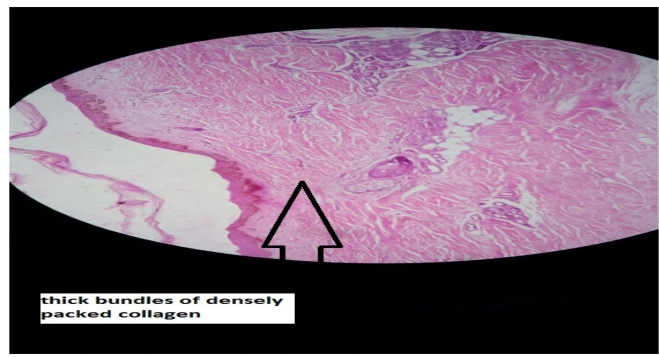

Picture 3: Skin biopsy of the child revealing increased collagen bundles

The child was encouraged to be as physically active as possible. General skin care which included avoiding irritating or drying substances and the daily application of water soluble cream as an emollient was advised. The parents were told to avoid cold, trauma, heat and sun exposure as cold and trauma could exacerbate the symptoms. 


\section{Discussion}

Systemic sclerosis ( $\mathrm{SSc}$ ) is a rare but potentially lifethreatening condition. There are three main clinical subtypes associated with different morbidities: diffuse cutaneous (dc) SSc, limited cutaneous SSc (lcSSc), characterized by restricted and non progressive skin thickening (limited to distal extremities) associated with late visceral disease, and overlap SSc. Patients with CREST syndrome (calcinosis, Raynaud's phenomenon, esophageal dysmotility,sclerodactyly,and telangiectasia) are considered to have lcSSc. Some patients with undifferentiated or mixed connective tissue disease may have shiny or full appearing skin of the distal fingers, but they must have the major criterion, skin induration or sclerosis proximal to the metacarpal (MCP) or metatarsophalangeal (MTP), to be considered to have juvenile SSc. There are an additional two minor criteria required for the diagnosis of SSc in children according to international consensus agreement [1].

The minor criteria include cutaneous involvement (sclerodactyly), peripheral vascular involvement (Raynaud's pheno-menon, nailfold capillary abnormalities, digital tip ulcers), gastrointestinal, cardiac (arrhythmias, heart failure), renal (new onset renal hypertension, renal crisis), respiratory (pulmonary fibrosis, pulmonary artery hypertension), neurologic (neuropathy, carpal tunnel syndrome) and musculoskeletal system involvement. Serology positivity for antibodies is also included in among the minor criteria [5].

Juvenile systemic sclerosis is a rare connective tissue disease of unknown etiology. It is one of the most important rheumatic diseases in childhood. Fibrosis of the skin, subcutaneous tissue and internal organs are some of the characteristic features. Therefore, early detection of the severity of the disease may play a role in establishing an effective therapeutic regimen.

The serologic tests include a positive test for antinuclear antibodies, SSc selective antibodies (anticentromere, anti- topoisomerase 1 ( $\mathrm{Scl} 70)$, anti-fibrillarin, anti PM Scl, anti-fibrillin or anti RNA polymerase 1or 111) [5].

Skin biopsies from patients with scleroderma show various histologic features. Characteristics are particularly evident in the dermal microvasculature, including endothelial swelling and concentric thickening of the vascular basement membrane [6].

Multiple organ involvement in children at the time of diagnosis may be less common. The majority of the children present with changes of the hands and feet and/ the Raynaud's phenomenon. Other skin manifestations may be less frequent such as sclerodactly, edema and calcinosis [7]. The renal, cardiac and gastrointestinal systems are involved. Arthralgia and myalgia, typically the morning stiffness can be present [7].

The treatment for scleroderma varies according to the subtype and severity. Superficial morphea may benefit from topical corticosteroids or ultraviolet therapy. For lesions involving deeper structures, systemic therapy is recommended. The treatment plan for Juvenile Localized Scleroderma (JLS) includes 1: subcutaneous methotrexate given at $1 \mathrm{mg} / \mathrm{kg}$ weekly (maximum dose: $25 \mathrm{mg}$ ) 2: weekly methotrexate plus either three months of high dose of intravenous corticosteroids for 3 consecutive days a month or 3: high daily oral corticosteroids $(2 \mathrm{mg} / \mathrm{kg} /$ day; maximum: $60 \mathrm{mg}$ ) slowly tapered over 48 weeks [8].

Treatment of Juvenile Systemic Sclerosis targets specific disease manifestations. Raynaud's phenomenon is treated mainly by cold avoidance and nifedipine (30$60 \mathrm{mg}$ sustained released form) is reserved for the severe form. If there is hypertension associated with renal involvement then the use of angiotensin converting enzyme inhibitors are recommended.

Skin manifestations benefit from the use of methotrexate and mycophenolate mofetil. Cyclophosphamide and mycophenolate mofetil have been used to treat pulmonary alveolitis and to prevent pulmonary fibrosis [8].

Management approaches appropriate for pediatric patients should be chosen considering any possible adverse effects of the medications (corticosteroid induced growth failure) as well as the psychosocial impact of the chronicity and physical deformity for both the child and the parents.

\section{Conclusion}

A detailed history and keen examination is important not to miss a connective tissue disorder. Physicians and patients should be more attentive to the potential risk factors for organ damage particularly very early in the disease, even when the children may not be symptomatic. Early initiation of treatment could go a long way and lead to a better quality of life.

Funding: Nil, Conflict of interest: None initiated, Perission from IRB: Yes 


\section{References}

1. Torok KS (2012) Pediatric scleroderma: systemic or localized forms. Pediatr Clin N Am 59:381-405. DOI: https://doi.org/10.1016/j.pcl.2012.03.011.

2. Scalapino K, Arkachaisri T, Lucas M, Fertig N, Helfrich DJ, Londino AV, Jr, Steen VD, Medsger TA., Jr Childhood onset systemic sclerosis: classification, clinical and serologic features, and survival in comparison with adult onset disease. J Rheu-matol. 2006;33(5):1004-1013.

3. Martini G, Foeldvari I, Russo R, Cuttica R, Eberhard A, Ravelli A, Lehman TJ, de Oliveira SK, Susic G, Lyskina G, et al. Systemic sclerosis in childhood: clinical and immunologic features of 153 patients in an international database. Arthritis Rheum. 2006;54(12): 3971-3978. DOI: https://doi.org/10.1002/art.22207

4. Zulian F, Athreya BH, Laxer R, Nelson AM, Feitosa de Oliveira SK, Punaro MG, Cuttica R, Higgins GC, Van Suijlekom-Smit LW, Moore TL, et al. Juvenilelocalized scleroderma: clinical and epidemiological features in 750 children. Aninternational study. Rheu-matology (Oxford) 2006; 45(5): 614-620.DOI: https: // doi.org/10.1093/ rheumatology/ kei251

5. A Yadav, TP Yadav, V Gupta. Juvenile Systemic Sclerosis. Journal of Indian Academy of Clinical Medicine, 2011; 12( 2): 128-33

6. Fleischmajer R, Perlish JS, Reeves JRT. Cellular infiltrates in scleroderma skin. Arthritis Rheum1977; 20:975-984.doi:https://doi.org/10.1002/art. 1780200410

7. Nietert PJ, Sutherland SE, Silver RM, et al. Is occupational organic solvent exposure a risk factor for scleroderma? Arthritis Rheum 1998; 41: 1111-18: doihttps: //doi. org/10.1002/1529-0131(199806)41: 6<1111:: AID-ART19>3.0.CO;2-J

8. Robert M, Kleigman, Bonita, Stanton, Joseph St.G, Schor NF. Neslon Textbook of Pediatrics. $20^{\text {th }}$ edition. Philadelphia: Elsevier Health;2015

\section{How to cite this article?}

Shah Z, Mohan D.S, Parikh Y. Rapidly progressing juvenile scleroderma: A case report. Int J Pediatr Res. 2018;5(6): 339-342.doi:10.17511/ijpr.2018.i06.07. 